\title{
Spectral Anomaly Methods for Aerial Detection using KUT Nuisance Rejection
}

\author{
R. S. Detwiler, D. M. Pfund, M. J. Myjak, J. A. Kulisek, C. E. Seifert
}

Pacific Northwest National Laboratory, Richland, Washington, 99352, United States

Abstract

This work discusses the application and optimization of a spectral anomaly method for the realtime detection of gamma radiation sources from an aerial helicopter platform. Aerial detection presents several key challenges over ground-based detection. For one, larger and more rapid background fluctuations are typical due to higher speeds, larger field of view, and geographically induced background changes. As well, the possible large altitude or stand-off distance variations

11 cause significant steps in background count rate as well as spectral changes due to increased

12 gamma-ray scatter with detection at higher altitudes. The work here details the adaptation and

13 optimization of the PNNL-developed algorithm NSCRAD (Nuisance-Rejecting Spectral

14 Comparison Ratios for Anomaly Detection), a spectral anomaly method previously developed

15 for ground-based applications, for an aerial platform. The algorithm has been optimized for two

16 multi-detector systems; a NaI(Tl)-detector based system and a CsI detector array. The

17 optimization here details the adaptation of the spectral windows for a particular set of target

18 sources to aerial detection and the tailoring for the specific detectors. As well, the methodology

19 and results for background rejection methods optimized for the aerial gamma-ray detection using

20 Potassium, Uranium and Thorium (KUT) nuisance rejection is shown. Results indicate that use

21 of a realistic KUT nuisance rejection may eliminate metric rises due to background magnitude 
1 and spectral steps encountered in aerial detection due to altitude changes and geographically

2 induced steps such as at land-water interfaces.

3 Keywords: Spectral Anomaly Detection, KUT Nuisance Rejection, Aerial Search, Aerial

4 Detection, Radiation Detection

\section{$5 \quad 1.0$ Introduction}

6 Aerial detection combines all the challenges of standoff detection with all the difficulties of

7 mobile survey, including large and varying standoff distances and abrupt and significant changes

8 in background. Critical issues for aerial detection include land-water interfaces, rapidly changing

9 KUT backgrounds, and varying down scatter with altitude changes. To address these issues a

10 spectral anomaly method has been adapted to aerial detection. The NSCRAD (Nuisance-

11 Rejection Spectral Comparison Ratio) algorithm was initially developed at PNNL for medium

12 resolution detectors [1]. The algorithm has been used for a range of ground-based detection

13 applications, including ground-based detection, portal monitoring, and OSI applications

$14[2,3,4,5]$. The method used in NSCRAD includes both optimized energy windows for detection

15 of threat sources and a nuisance rejection capability. The spectral anomaly method was

16 optimized in this work for aerial detection systems to increase the probability of detection of

17 threat sources in aerial search while using the nuisance rejection capability to minimize false

18 alarms from rapidly changing aerial background. The implementation of NSCRAD for aerial

19 detection requires optimizing energy windows, adapting KUT nuisance rejection for aerial

20 backgrounds, developing new methods for background tracking, and adjusting tracking and

21 detection thresholds.

\section{$22 \quad 2.0$ Methodology}


2 Previous methods have been developed for detection of anomalies in spectra [6]. The specific

3 methodology used here by NSCRAD involves the following steps [1,7]. First, spectral windows

4 are optimized for a given set of threat and nuisance sources by maximizing the differences

5 between these categories as measured by the windows [7]. The process involves both

6 optimization of detection of the threat sources and rejection of the background and nuisance

7 sources [8]. Nuisance sources typically include background components ${ }^{40} \mathrm{~K},{ }^{238} \mathrm{U}$ and ${ }^{232} \mathrm{Th}$

8 (KUT) and progeny, but may also include medical or industrial sources. An example set of

9 windows is shown in Figure 1.

10 Next, the vector of background-scaled spectral comparison ratios (SCRs) is calculated for each

11 spectra or sample time. The SCR's are count rate differences between spectral windows, scaled

12 by the mean backgrounds, as shown here:

$$
c_{\alpha(1,2)}=c_{1}-\frac{\mu_{1}}{\mu_{2}} c_{2}
$$

14 where $\mu$ is the mean background and the numerals in the subscripts refer to the different spectral

15 windows. Next, vector SCRs are calculated for the Naturally Occuring Radioactive Material

16 (NORM) and assumed nuisance spectra, and these components are removed from the sample

17 spectra using an orthogonal subspace projection:

$$
\mathbf{P}_{\alpha}=\mathbf{A}_{\alpha}\left(\mathbf{A}_{\alpha}^{T} \boldsymbol{\Sigma}_{\alpha}^{-1} \mathbf{A}_{\alpha}\right)^{-1} \mathbf{A}_{\alpha}^{T} \boldsymbol{\Sigma}_{\alpha}^{-1}, \quad \mathbf{A}_{\alpha}=\left[\begin{array}{llll}
\mathbf{a}_{\alpha 1} & \mathbf{a}_{\alpha 2} & \mathbf{a}_{\alpha 3} & \mathbf{a}_{\alpha 4}
\end{array}\right]
$$

Here, $\sum$ is the background covariance. Finally, the standardized distance metric is calculated,

20 and weighted by background covariance as shown below; an observed value above a given

21 threshold, D, indicates threat or anomalous source. 


$$
D=\sqrt{\mathbf{c}_{\alpha}^{T} \boldsymbol{\Sigma}_{\alpha}^{-1}\left(\mathbf{c}_{\alpha}-\mathbf{P}_{\alpha} \mathbf{c}_{\alpha}\right)}
$$

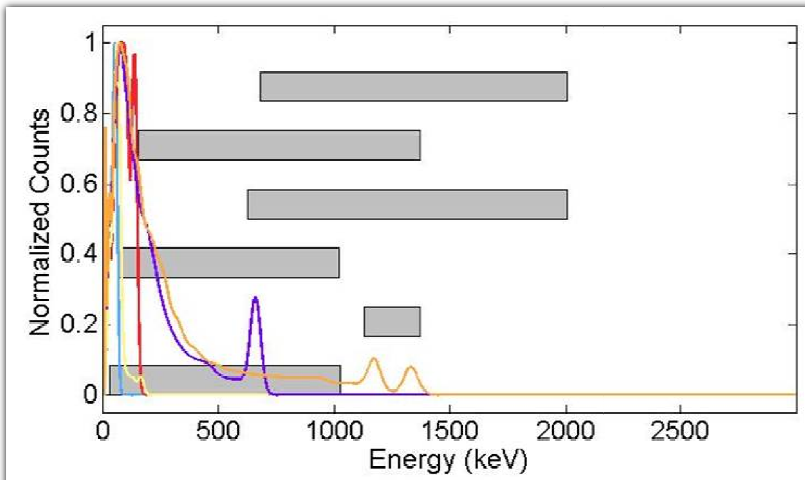

Am-241, Tc-99m, TI-201, Cs-137 and Co-60

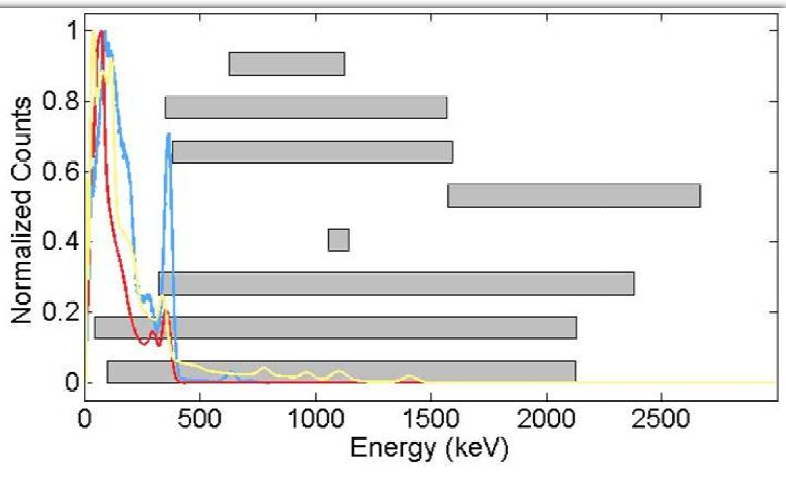

I-131, Ba-133, Eu-152
2

4 rejection.

\subsection{General Approach}

The spectral anomaly method NSCRAD was optimized for two helicopter-based gamma-ray

7 detection systems using $\mathrm{NaI}(\mathrm{Tl})$ and $\mathrm{CsI}(\mathrm{Na})$ detector arrays. The adaptation of the spectral anomaly method NSCRAD for aerial detector systems involves both optimization of the

9 background rejection methodology for aerial detection as well as optimization of parameters

10 related to source detection. An important factor in source detection, which varies with stand-off

11 distance and speed, is the integration time used in the algorithm; this time was optimized through

12 analysis using source injections to a value of 3 seconds. This factor agreed with past calculations

13 based on speed and offset [9]. The background rejection methodology and settings were

14 investigated using aerial background flyover data from a $\mathrm{NaI}(\mathrm{Tl})$ based detector system spanning

15 a large altitude range of $30 \mathrm{~m}$ to $400 \mathrm{~m}$ and involving two flight locations. We examined

16 whether the NSCRAD settings need to be adjusted with altitude and location. The spectral 
1 ROI's for a set of target sources were also optimized for the particular detection system using

2 simulated spectra at a typical flight altitude of $300 \mathrm{ft}$.

3 The results with re-optimization of windows and addition of background $\mathrm{K}$, $\mathrm{U}$ and Th nuisance

4 rejection based on the particular detector system showed a significant reduction in the

5 background-induced jumps in the NSCRAD metric corresponding to altitude changes. The

6 results also indicated that with realistic nuisance rejection and energy windows optimization, no

7 altitude dependent thresholds were needed.

8

9 Two established methods for background tracking with NSCRAD were adapted for the aerial

11 constant, based on the following equation:

12

$$
\hat{\mathbf{B}}_{k}=\frac{1}{60} \sum_{j=0}^{59} \mathbf{B}_{k-j}
$$

13 The second is the Exponentially Weighted Moving Average (EWMA):

14

$$
\hat{\mathbf{B}}_{k}=(1-\lambda) \hat{\mathbf{B}}_{k-1}+\lambda \mathbf{B}_{k}
$$

15 The exponential constant here is set to a value of 0.0443 to match the $50 \%$ power cutoff

16 frequency of the EWMA to that of a 60 s rolling average. The value of 60 seconds was chosen

17 from optimization studies using source injections on real flight background data for the $\mathrm{NaI}(\mathrm{Tl})-$

18 based detector system.

19 Detection and tracking thresholds were set using available flight data for the detector system, and

20 balancing the percent of false positives [9]. The data included two sets of flight data, in two

21 different geographic locations, spanning altitude ranges of $50 \mathrm{ft}$ above ground level (AGL) to 
1 600-700 ft AGL, with a combined total of over 10,000 data points. Below in Figure 2 is an

2 illustration of the method for one geographic location, of 9,300 data points. The method

3 illustrates the requirements of setting the alarm and tracking thresholds based the allowed

4 number of false positives. The metrics are shown for three sets of energy windows,

5 corresponding to specific low, medium and high energy sources.

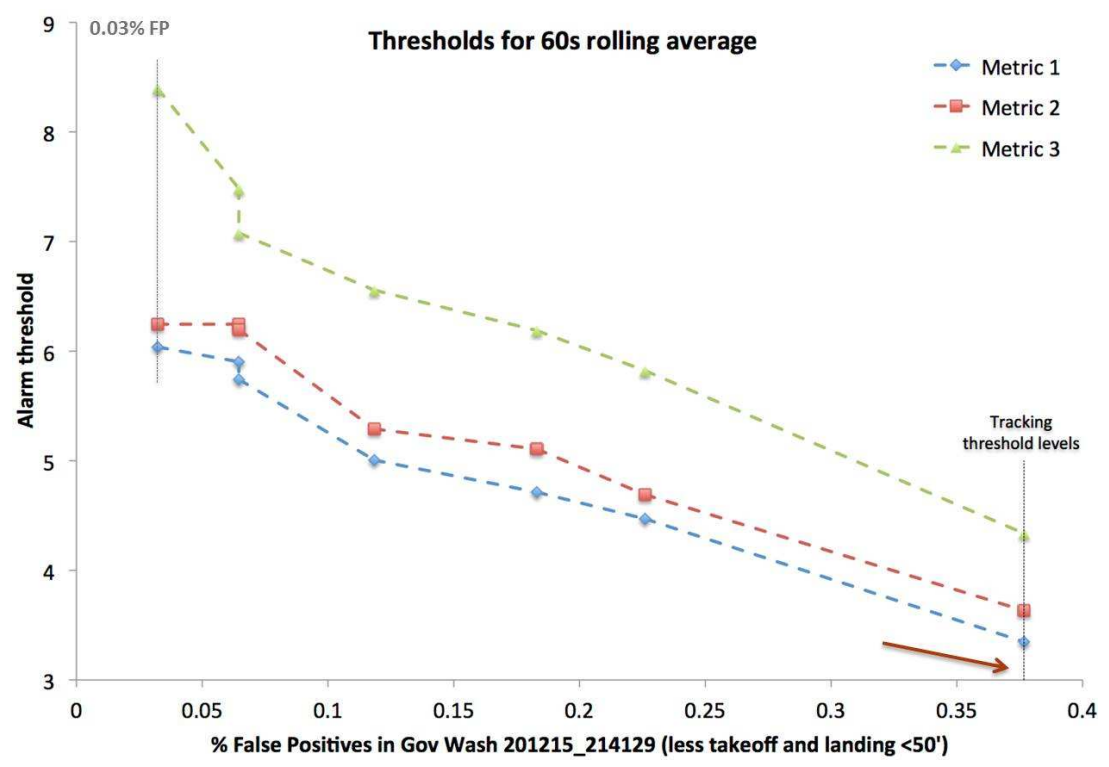

7 Figure 2. Balancing alarm thresholds vs. false positive rates for the rolling 60 second

8 background method.

\section{$9 \quad 3.0$ Results}

\subsection{Window Optimization}

11 A comparison of the NSCRAD metric before and after the adapted KUT background rejection

12 and window optimization for a particular set of energy for detection of ${ }^{133} \mathrm{Ba}$ and ${ }^{131} \mathrm{I}$ is shown in

13 Figure 3. Here, results on the left use generic energy windows for large $\mathrm{NaI}(\mathrm{Tl})$ detector

14 geometries from land-based applications. Results on the right use energy windows which are 
1 optimized for a similar detector geometry, but for aerial applications. The new windows were

2 developed using the adjusted detector response function based on air attenuation and scatter,

3 using real flight data from the $\mathrm{NaI}(\mathrm{Tl})$-based aerial detector system at a range of flying altitudes.

4 NSCRAD metric results from real flight data from this system are shown below, using previous

5 generic land-based windows on the left and windows optimized for the aerial detector system on

6 the right. This data set included several real medical/industrial sources as indicated by the

7 elevated metrics in the figure; these are detected by both sets of parameters. However, other

8 metric rises shown in the left figure are associated with altitude or background changes; these are

9 reduced or eliminated with the addition of the KUT nuisance rejection and re-optimized energy

10 windows. In addition, an important result from adaptation of windows for aerial detector

11 response function is that since the metric rises from background and altitude changes are

12 minimized with aerial energy windows, altitude-dependent thresholds are not required.

13

14

15 for aerial applications (right), as a function of altitude for data from an urban/maritime area and a
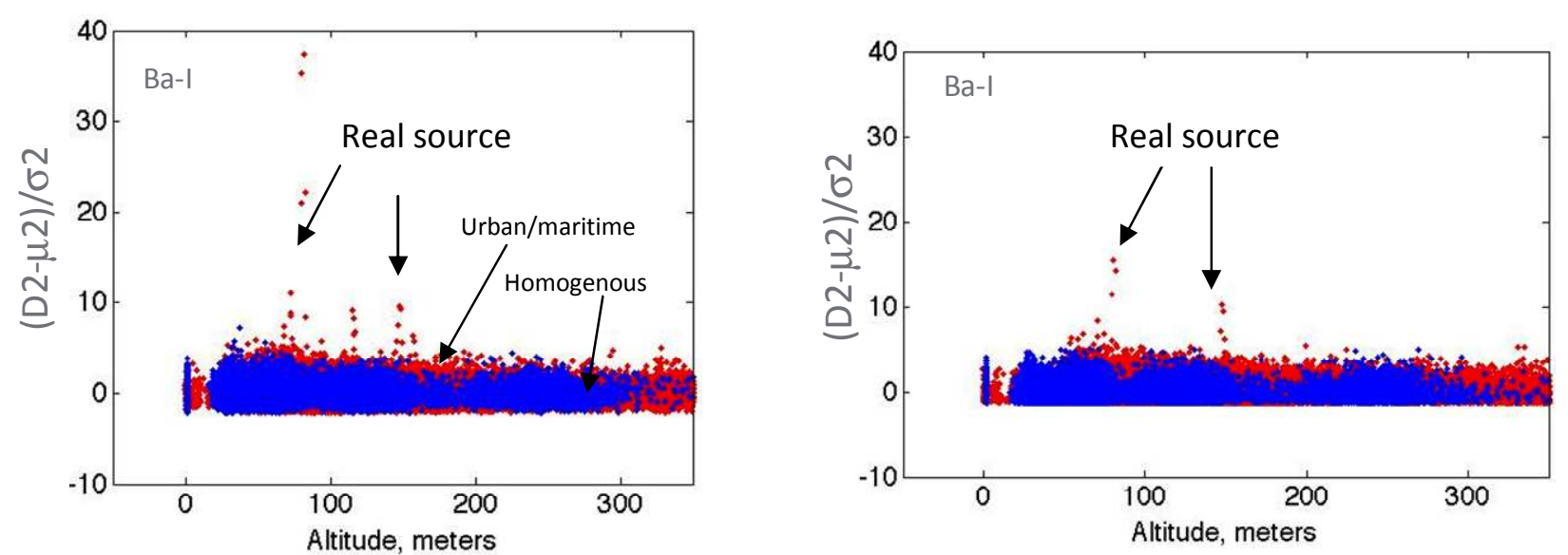

Figure 3. Comparison of NSCRAD Metrics without optimization (left) and with optimization relatively homogeneous background area. 
1 An example showing the importance of adapting spectral anomaly windows for the detector

2 system geometry specifications is shown below. Here, analysis is shown for simulated flight

3 data using simulated background, with source injections, as has been done with past NSCRAD

4 sensitivity comparisons [9,10]. Source injections included Cs-137 and Co-60 source of varying

5 strengths. Results in Figure 4 show a comparison of the analysis with two sets of windows, both

6 optimized for aerial detection at similar altitude ranges, but for different detector geometries.

7 Windows on the left are based on a $\mathrm{NaI}(\mathrm{Tl})$ array of large crystals, while windows on the right

8 are based on a CsI array with smaller detector geometries.

9 The simulated background was developed using measured ratios of KUT components from flight

10 data form the $\mathrm{NaI}(\mathrm{Tl})$ based detector system over a relatively homogenous background. The

11 background spectral response was simulated for a new detector geometry, based on an array of

12 CsI detectors of smaller detector geometries, using the measured KUT concentrations. Next,

13 simulated second-by-second flight data was developed using simulated background spectra with

14 Poisson variation added, and injections of source spectra were made using simulated detector

15 responses for a particular flight altitude and source offset. Figure 4 shows a comparison of the

16 analysis with two sets of windows, at a flight altitude of $300 \mathrm{ft}$ AGL, for both zero and $300 \mathrm{ft}$

17 offsets. There is an approximately $30 \%$ gain in sensitivity, taken as the ratio of the source metric

18 to the average background, using the energy windows adapted to new detector geometry. 

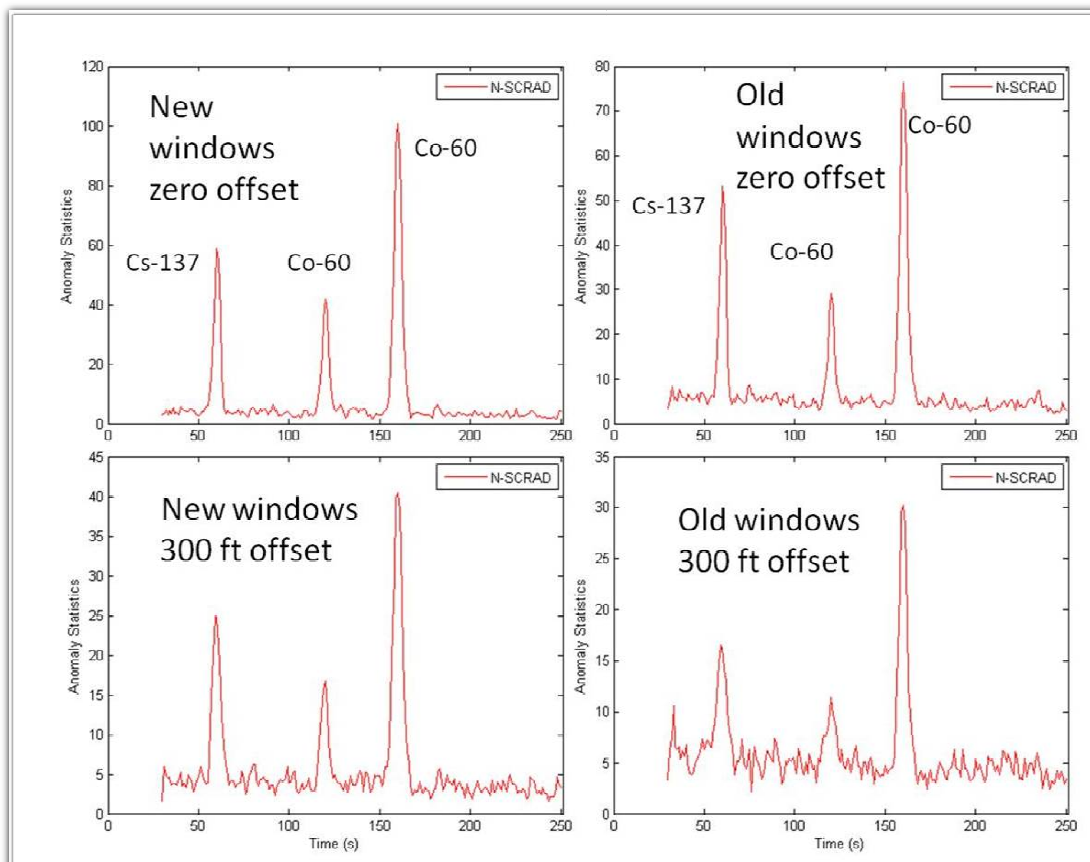

1

Figure 4. Comparison of NSCRAD metric results using aerial windows optimized for a CsIbased detector geometry (left) and aerial $\mathrm{NaI}(\mathrm{Tl})$-based windows (right) using simulated flight data for the CsI-based detector system.

\subsection{KUT Background Rejection Optimization}

\subsubsection{Analysis of Performance with Artificial Background Steps}

As part of the optimization for aerial detection, analysis involving real and induced background magnitude and spectral steps, such as those encountered at a land-water interface, was conducted. Initial analysis involved artificially induced background steps of both count rate and of an individual background spectral component. This analysis allowed a controlled evaluation of performance of the NSCRAD background rejection capabilities. Here, background flight data was generated for the 12 detector $\mathrm{NaI}(\mathrm{Tl})$ system using simulated background spectra from

measured KUT concentrations at $100 \mathrm{ft}$ AGL for an area of relatively homogeneous background, 
with Poisson variation added. NSCRAD analysis was conducted using the two background

2 methods. For both methods, background tracking thresholds were set for the NaI(Tl)-based

3 detector system using real flight data. Metric results from a $5 \mathrm{X}$ background step in count rate

4 only is shown in Figure 5; here the relative background spectral components are kept constant

5 (with addition of Poisson variation). The results without nuisance rejection are labeled as

6 SCRAD; results with nuisance rejection are labeled as NSCRAD. A correction to the covariance

7 matrix based on the gross count rate was also applied for these results [9]. Metric results are

8 scaled by a detection threshold set for the $\mathrm{NaI}(\mathrm{Tl})$ system from real flight data, with a value of

9 one corresponding to a detection. The results show some sensitivity to the background step

10 without nuisance rejection, with some difference in this example between the two background

11 methods. However, for both methods, NSCRAD results (with nuisance rejection) reduce the

12 metric at the spectral step to a value at or below the detection threshold.

13 Metric results from a 5 X step in an individual spectral component are shown in Figures 6 and 7;

14 here a single spectral component is varied, while keeping the background count rate constant.

15 Results from steps in K-40 have a close similarity in the SCRAD results (without nuisance

16 rejection) between background methods (Figure 6). Steps in ${ }^{238} \mathrm{U}$ and ${ }^{232} \mathrm{Th}$ (Figure 7) show

17 some differences in the SCRAD responses between background methods. However, in all cases

18 the SCRAD response show sensitivity to the background step, with a metric above the detection

19 threshold, as the step differs spectrally from the background. In all cases the NSCRAD metric

20 (with KUT nuisance rejection) shows a similar response for both methods, and a significant

21 reduction in the metric at the step, reducing the metric to below the detection threshold. 

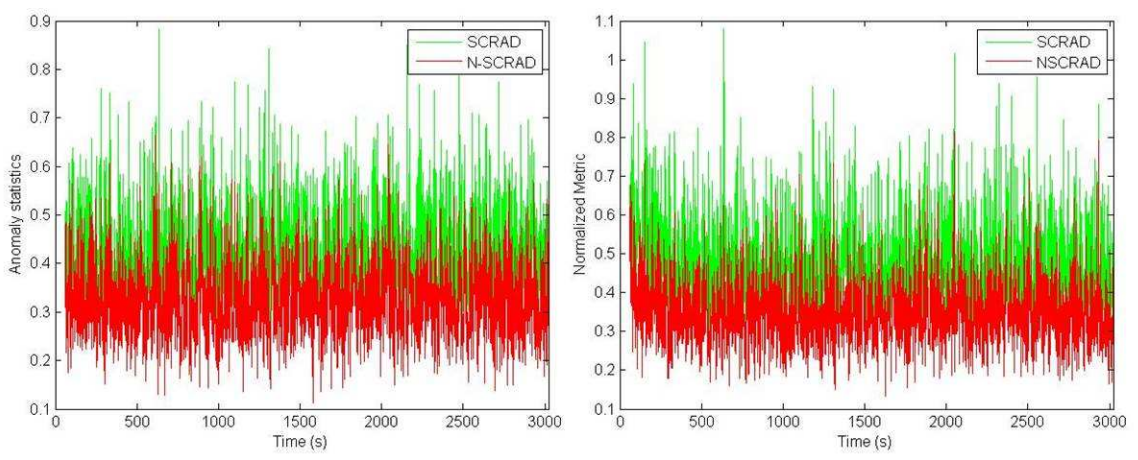

1

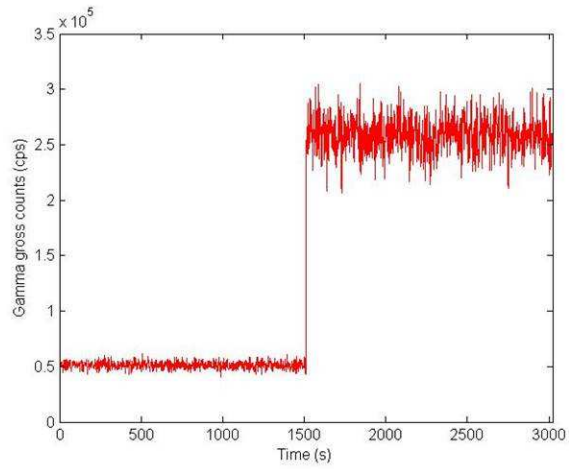

2 Figure 5. Results with and without nuisance rejection for an artificially induced $5 \mathrm{X}$ count rate 3 step.

4
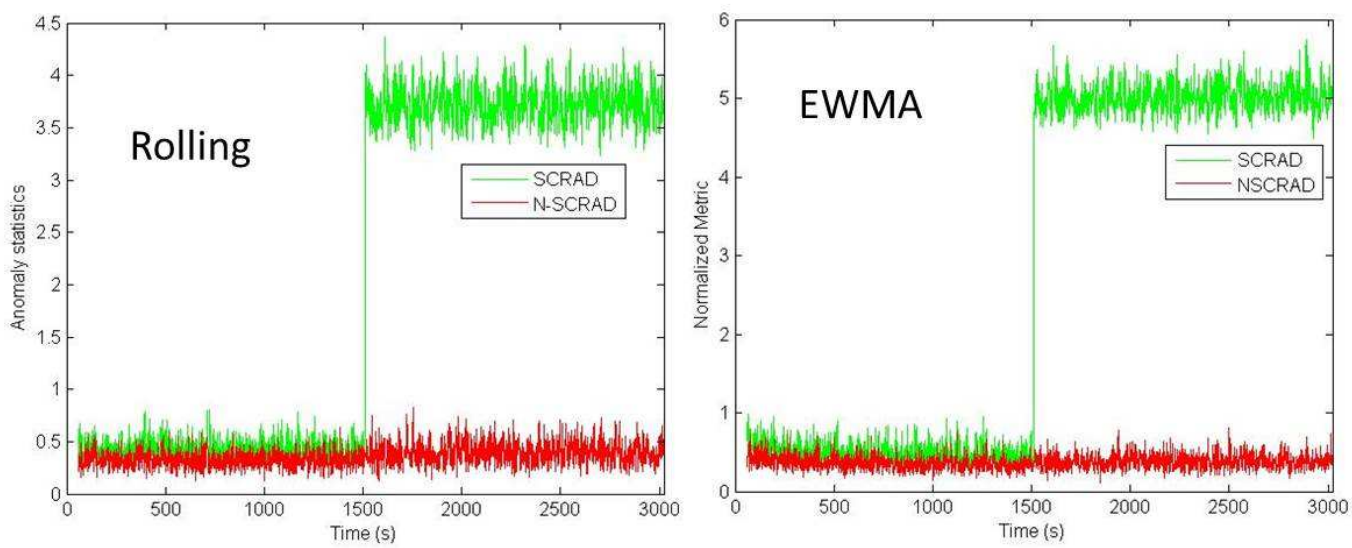

$5 \quad$ Figure 6. Results with and without nuisance rejection for an artificially induced 5X spectral step 6 in K-40. 

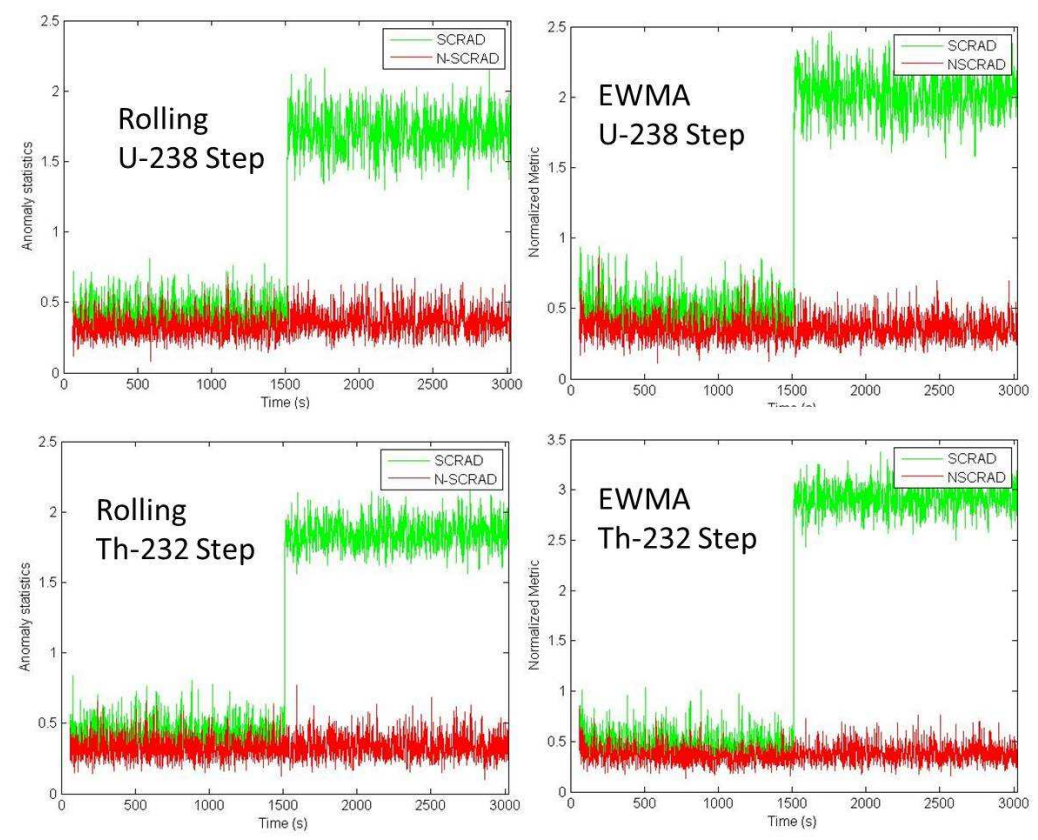

2 Figure 7. Results with / without nuisance rejection for $5 \mathrm{X}$ spectral step in ${ }^{238} \mathrm{U}$-and ${ }^{232} \mathrm{Th}$.

\subsubsection{Background Tracking Thresholds}

4 As described in section 2.2, the above analysis used a background tracking threshold that was optimized for the $\mathrm{NaI}(\mathrm{Tl})$-based detector system. Figure 8 illustrates the effect on varying

6 tracking thresholds on the SCRAD and NSCRAD metrics. The responses for the EWMA

7 background tracking method are shown for a $5 \mathrm{X}$ step in the ${ }^{238} \mathrm{U}$ background component, for

8 three sets of thresholds. Results are shown for the optimized threshold, one-half and $2 \mathrm{X}$ the

9 threshold. It is apparent that lower tracking thresholds increase the SCRAD response to the

10 induced background step, while higher thresholds reduce the response to the step. However,

11 higher thresholds may also reduce the NSCRAD responses to real sources. As seen, for low,

12 optimum and high tracking thresholds, the NSCRAD response (with nuisance rejection) does

13 significantly reduce the metric value at the background step. 

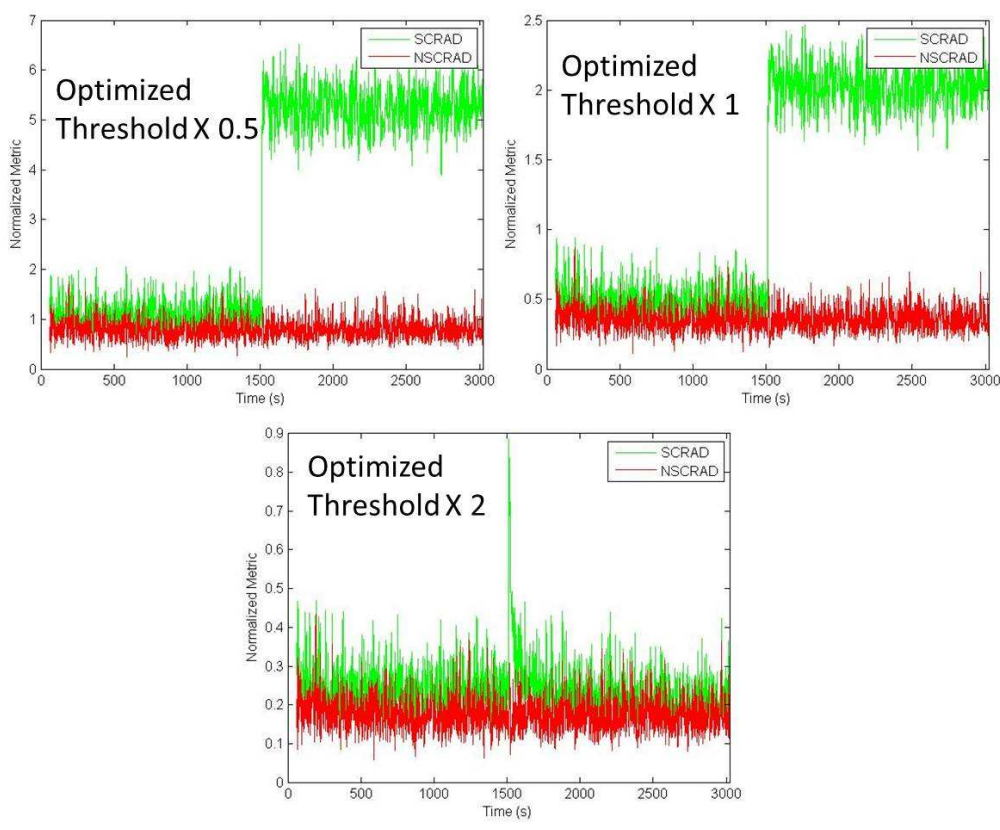

2 Figure 8. Effects of Background tracking Threshold on SCRAD/NSCRAD Response for 5X 3 Step in ${ }^{238} \mathrm{U}$.

\subsubsection{KUT Rejection with Land/Water Interface}

5 An example of the background rejection response of the algorithm to real flight data over a

6 land/water interface using optimized KUT nuisance rejection is shown in Figure 9. The flight

7 data, with gross count rates shown at the left, is from a 12-detector $\mathrm{NaI}(\mathrm{Tl})$ array, and contained

8 multiple count rate jumps due to repeated passes over the land-water interface. The NSCRAD

9 metric response is shown on the right, using the two background methods mentioned above, with

10 EWMA in red and the Rolling sum in blue, for a set of spectral windows for high energy sources.

11 The absolute metric is shown (not scaled by the detection threshold). Both background methods

12 produce similar results using KUT nuisance rejection. As well, the metric is relatively flat over

13 the large steps in count rate, and less than a typical detection threshold of 7-8 for all background 14 jumps. 


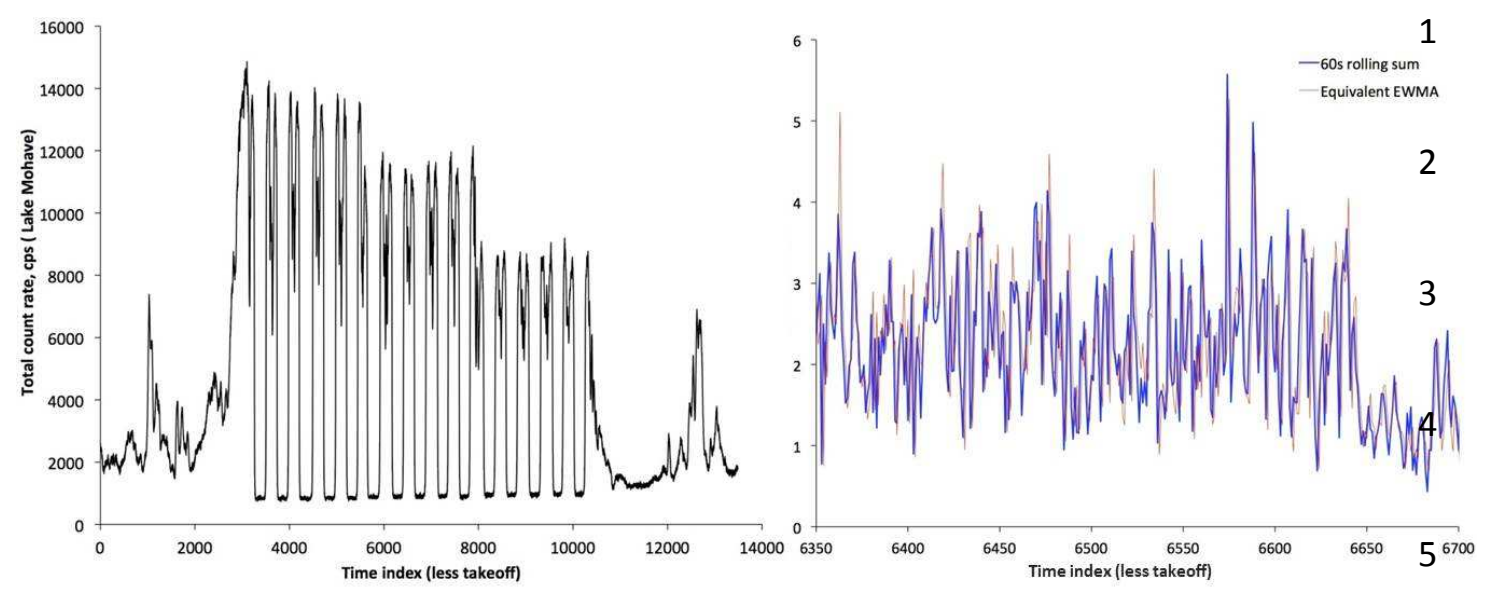

6 Figure 9. Lake Mohave flight data showing total count rate (left) with NSCRAD Metric results

7 for land-water interface.

\section{Conclusion}

9 The spectral anomaly methods described here, with nuisance rejection, have demonstrated the

10 capability for adaptation to aerial detection. The optimization process includes adjustment of

11 energy regions or windows for a particular set of threat and nuisance sources, based on the given

12 detector response function. It was demonstrated that optimization of windows is needed both for

13 differences in application, such as ground-based detection vs. aerial detection, as well as

14 different detector geometries. Results with energy windows optimized for a specific aerial

15 detection system show the ability for improved detection of threat sources and rejection of

16 background-induced false-alarms encountered in aerial detector search applications.

17 As well, the optimization of KUT nuisance rejection for a given aerial detector system shows

18 promise for improved rejection of the false alarms caused by sharp steps in background. This

19 optimization was demonstrated with real flight data over a land/water interface, which included

20 both sharp count rate steps and spectral changes. The results showed that with adapted KUT

21 nuisance rejection, the steps were largely eliminated and remained below the detection 
1 thresholds. The addition of KUT rejection with optimized energy windows also showed a

2 reduction in the effects of altitude changes during flight, eliminating the need for altitude specific

3 thresholds. Future work will investigate the dependence of energy windows KUT nuisance

4 rejection on specific backgrounds for aerial detection, to determine if sensitivity improves with

5 use of background-specific windows in certain scenarios such as a land/water interface.

\section{Acknowledgements}

7 This work has been supported by the US Department of Homeland Security, Domestic Nuclear

8 Detection Office, under competitively awarded contract/IAA HSHQDC-12-X-00376. This

9 support does not constitute an express or implied endorsement on the part of the Government.

10 This report is PNNL-SA-103907. Pacific Northwest National Laboratory is operated for the

11 U.S. Department of Energy by Battelle under Contract DE-AC05-76RL01830.

\section{References}

13 [1] D. M. Pfund, R. C. Runkle, K. K. Anderson, and K. D. Jarman, "Examination of Count-

14 Starved Gamma Spectra Using the Method of Spectral Comparsion Ratios," IEEE Trans. Nucl.

15 Sci., vol. 54, no. 4, pp. 1232-1238, Aug. 2007.

16 [2] R. C. Runkle, M. J. Myjak, S. D. Kiff, D. E. Sidor, S. J. Morris, J. S. Rohrer, K. D.

17 Jarman, D. M. Pfund, L. C. Todd, R. S. Bowler, and C. A. Mullen, "Lynx: An unattended sensor

18 system for detection of gamma-ray and neutron emissions from special nuclear materials," Nucl.

19 Instrum. Methods Phys. Res. A, vol. A598, pp. 815-825, 2009. 
1 [3] R. C. Runkle, M. F. Tardiff, K. K. Anderson, D. K. Carlson, and L. E. Smith, “Analysis

2 of spectroscopic radiation portal monitor data using principal components analysis," IEEE Trans.

3 Nucl. Sci., vol. 53, no. 3, pp. 1418-1423, Jun. 2006.

4 [4] R. C. Runkle, T. M. Mercier, K.K. Anderson, and D. K. Carlson, "Point source detection 5 and characterization for vehicle radiation portal monitors," IEEE Trans. Nucl. Sci., vol. 52, no. 6, 6 pp. 3020-3025, Dec. 2005.

7 [5] Carolyn E. Seifert, Mitchell J. Myjak, and David M. Pfund Pacific Northwest National 8 Laboratory, "Detection of Anomalous Gamma-Ray Spectra for On-Site Inspection," The 9 International Scientific Studies Project 2009 Conference Proceedings (ISS 2009).

[6] B. Ng, Survey of Anomaly Detection Methods Lawrence Livermore National Laboratory, 11 Livermore, CA, Tech. Rep. UCRL-TR-225264, Oct. 2006.

12 [7] K. K. Anderson, K. D. Jarman, M. L. Mann, D. M. Pfund, R. C. Runkle, “Discriminating 13 nuclear threats from benign sources in gamma-ray spectra using a spectral comparison ratio 14 method," Journal of Radioanalytical and Nuclear Chemistry, vol. 276, no.3 (2008) 713-718.

15 [8] J. H. Ely, R. T. Kouzes, J. E. Schweppe, E. R. Siciliano, D. M. Strachan, and D. R. 16 Weier, "The use of energy windowing to discriminate SNM from NORM in radiation portal 17 monitors," Nucl. Instrum. Methods Phys. Res. A, vol. A560, pp. 373-387, May 2006.

18 [9] David Michael Pfund, Kenneth D. Jarman, Brian D. Milbrath, Member, IEEE, Scott D. 19 Kiff, Member, IEEE, and Daniel E. Sidor, "Low Count Anomaly Detection at Large Standoff 20 Distances," IEEE Trans. Nucl. Sci., vol. 57, no. 1, Feb. 2010. 
1 [10] Kenneth D. Jarman, Robert C. Runkle, Kevin K. Anderson, David M. Pfund, “A

2 comparison of simple algorithms for gamma-ray spectrometers in radioactive source search

3 applications," Applied Radiation and Isotope, vol. 66 pp. 362-371, 2008. 